\title{
Transparent and Biocompatible Electrodes Based on Carbon Nanotubes/Albumin Composite
}

\author{
Dmitry Kireev, Ivan I. Bobrinetskiy ${ }^{*}$, Alexey S. Seleznev, Igor V. Fedorov, Alexey V. Romashkin, \\ Roman A. Morozov
}

Center for Probe Microscopy and Nanotechnology, National Research University of Electronic Technology, Moscow, Russia. Email: ${ }^{*} v k n @$ nanotube.ru

Received January $27^{\text {th }}, 2013$; revised February $28^{\text {th }}, 2013$; accepted March $25^{\text {th }}, 2013$

Copyright (C) 2013 Dmitry Kireev et al. This is an open access article distributed under the Creative Commons Attribution License, which permits unrestricted use, distribution, and reproduction in any medium, provided the original work is properly cited.

\begin{abstract}
We describe a new method for transparent and conductive films based on carbon nanotubes and bovine serum albumin composite development. Films are deposited from an aqueous solution of carbon nanotubes/bovine serum albumin by drop-coating and rod-coating methods. Sheet resistances of as-prepared films vary from $200 \mathrm{Ohm} / \mathrm{sq}$ with $50 \%$ transmittance to $30 \mathrm{KOhm} / \mathrm{sq}$ with $90 \%$ transmittance. The maximum $\sigma_{d c} / \sigma_{o p}$ ration found in this work is 2.27 , which gives a DC conductivity of $4.55 \times 10^{4} \mathrm{~S} \cdot \mathrm{m}^{-1}$. Atomic force microscopy and Raman spectroscopy studies of the films show that the process of film formation produces neither structural nor chemical changes in the nanotubes. Possibility of using these films for cell culturing is tested on human embryonic fibroblast cell line. Therefore, it is first time ever in literature, when proposed a method, allowing fabricating at the same time transparent, high-conductive and biocompatible CNT films.
\end{abstract}

Keywords: Carbon Nanotubes; Transparent Electrodes; Biocompatible Composite

\section{Introduction}

Flexible electrodes are necessary components of many current devices, including touchscreens [1], liquid-crystal displays [2], flexible displays [3], organic light emitting diodes [4] and solar cells [5]. Conventionally, such electrodes are made with conducting metal-oxide sputtering techniques. The most abundant of the compounds usedis Indium Tin Oxide (ITO) [6]. However, ITO has several drawbacks such as low mechanical strength, low conductivity, and as a consequence a high-energy consumption. Besides that it uses complex and environmentally hazardous chemical processes in production [7]. In addition, global reserves gradually running out of ITO, which increases the cost of this material. Moreover, ITO is not suitable for fundamentally new implantable electronic devices. All this encourages scientists to search for new materials for replacement.

One alternative group of materials that fit the purpose of flexible, transparent and conductive electrode production is carbon nanomaterials (graphene, carbon nanotubes) [8]. There are many articles and reviews about the de-

\footnotetext{
"Corresponding author.
}

velopment of thin conductive films of carbon nanotubes (CNTs) in the scientific literature, describing a range of different methods [9]. Some cases require using CNTs with surfactants in aqueous solutions [10], or CNT-alcohols solutions [11], CNT-acid solutions [12], etc. Surfactants here separate CNTs from each other: as they are amphiphilic molecules, surfactants bind to CNTs and help them to become soluble in water. Moreover, they prevent coagulation and agglomeration of CNT into bundles. Such solutions can stay homogeneous for several weeks and even months.

One of the problems tackled by conductive film creation is the development of biocompatible electrodes for research into cells proliferation activity [13] and their viability, for example, in nerve tissue regeneration [14]. Biocompatibility is a serious problem facing the use of CNT films in advanced biotechnology. In literature is noted that CNTs, while in aqueous solution with various surfactants such as SDS and SDBS are toxic to astrocytroma cells since the surfactants are toxic substances themselves [15]. Also, in the work of Shin and colleagues they treat CNTs with $\mathrm{HNO}_{3}$ [16], Parekh et al. are using $\mathrm{COCl}_{2}$ [17], and Hetch et al. the films are 
deposited from a chlorosulfonic superacid solution [11]: this definitely restricts the bio-compatibility of the films.

Another major limiting factor from a technical perspective against the usage of CNT films in large amounts is DC conductivity $\left(\sigma_{d c}\right)$ [11]. This work deals with the problem of increasing the conductivity of the biocompatible and transparent CNT films. The maximum value of DC conductivity in the work, of $\sigma_{d c}=4.55 \times 10^{4} \mathrm{~S} \cdot \mathrm{m}^{-1}$, corresponds to a film with $50 \mathrm{~nm}$ thickness and $50 \%$ transparency (at $550 \mathrm{~nm}$ ). The measured characteristics of prepared films may be worse than one can possible find in literature, but this was not the goal of our work $[16,17]$. The goal was to achieve biocompatible electrodes with optical and electronic characteristics enough to fabricate bio-implantable electronic devices.

\section{Materials and Methods}

\subsection{Materials and Films Preparation}

Materials: SWCNTs utilized in the work were prepared by arc-discharge method and provided by A. V. Krestinin (Institute of Problems of Chemical Physics of RAS) [18]. According to the manufacturer's estimation, the SWCNTs are of $95 \%$ purity and supplied as a CNT-paper. According to transmission microscopy, they are in the shape of bundles with diameters from 1 to $10 \mathrm{~nm}$. BSA powder, used in this work-is common and commercially accessible (Albumin Bovine Serum Fraction V, BioWest, P6152).

The solutions preparation: 0.1 mass $\%$ of BSA was added to distilled water followed by ultrasonic homogenisation [19]. Then CNTs was added to the solution, followed by a long duration intensive ultrasonication ( 8 10 hours, $50 \mathrm{~W}, 34 \mathrm{KHz}$ ). For the work we investigated the different CNT concentrations. The mass ratio of nanotube/albumin, 5/1,10/1 and 33/1 was prepared by dilution of initial 1/1 solution.

Films preparation: First of all, the surface (cover glass) accurately cleans in 2-propanol followed by ultrasound washing in 2-propanol solution. Then, as-prepared CNT-containing solution is deposited onto the surface by drop- or rod-coating. In the case of drop-coating method: the solution drops onto the substrate, followed by drying the drop. To accelerate the process, the substrate is exposed to incandescent light (5 - 10 minutes). Resultant films have a specific non-uniformity: they are thicker near the edges, and this creates a great irregularity in the electro-physical characteristics. In the case of rod-coating method: It is necessary by some means to overcome the problem of poor wetting and to achieve the better uniformity for the films and universality for the whole method. The role of such mean in the work plays a rod. Using rods, water was uniformly distributed over the whole substrate, thus wetting the surface. Consequently, thickness of the film decreases and uniformity increases. Otherwise the method is identical to the previous one. The last step of film manufacture is annealing. It is well known that BSA is denatured by heat treatment (BSA denatures already at $70^{\circ} \mathrm{C}-80^{\circ} \mathrm{C}$ ). But, in order to take into account the effect of CNTs on increasing the protein's thermal stability, the denaturation temperature could be raised [20]. Moreover, annealing contributes to the amelioration of the contacts between CNTs and the substrate or contact electrodes.

\subsection{Characterization}

Optical studies of prepared films were made on UVvisible light spectrophotometer (SP-2000, experimental design bureau "Spectr"). Electro-physical characteristics were measured in IPPP 1/5 (Semiconductor Parameters Meter; Ltd "MNIPI", Republic of Belarus) by 4-probe method. Film's topology was investigated by atomicforce microscope Solver P-47 (NT-MDT) in tapping mode with silicon cantilevers. Comparison of Raman spectra of pure BSA powder, albumin film and CNTBSA film is made on the complex confocal microscope/Raman spectrometer Centaur U HR ("Nanoscan technology" Ltd., Russia). For this purpose, the following two solutions were created: $0.5 \mathrm{mg} \mathrm{BSA}-1 \mathrm{ml} \mathrm{H}_{2} \mathrm{O}$ and $0.1 \mathrm{mg}$ SWCNT $-0.5 \mathrm{mg} \mathrm{BSA}-1 \mathrm{ml} \mathrm{H}_{2} \mathrm{O}$. The solutions were deposited onto the $\mathrm{Si}$ substrate with a thermal $\mathrm{SiO}_{2}$ layer, and $\mathrm{Au} / \mathrm{Cr}$ layers of $70 / 30 \mathrm{~nm}$ thicknesses were deposited.

\section{Results and Discussion}

\subsection{Uniformity of the Composite Films Prepared by Different Methods}

As mentioned above, there are plenty of different methods for the creation of thin, conductive CNT films [21]. Each method has its advantages and drawbacks. In our work we focus on creating a simple, easily scalable and most importantly-a biocompatible material.

In the work we describe two methods of films creation: drop-coating and rod-coating (Figure 1), which satisfy to followed requirements:

- Possibility of utilizing almost any CNT solution, but firstly, water-surfactant solutions;

- Scalability;

- Compatibility with most conventional microelectronics processes.
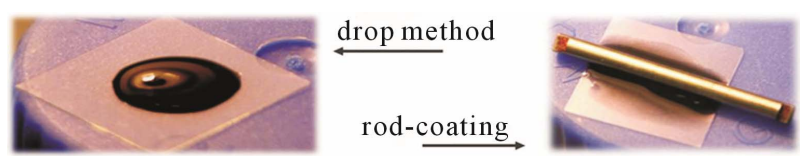

Figure 1. The schematics of drop-coating (left) and rodcoating (right) methods for CNT films preparation. 
The bovine serum albumin (BSA) plays the role of a surfactant. The albumin is a protein that commonly found in blood plasma. Accordingly to X-Ray crystallographic data, albumin structure is predominantly alpha-helical (67\%) [22].

BSA was chosen due to the fact that the CNT-BSA composite is, first of all, highly-stable and, second of all, biocompatible, which was demonstrated in a number of previous works $[23,24]$. Along with DNA, BSA appears to be a non-ionic surfactant in water solutions [25].

It was shown that drop-coated films are highly nonuniform, what was confirmed by AFM, optical studies and by dispersion of measured by four-probe technique conductivity values.

In this work, BSA is used as surfactant as soon as it allows making not only stable water solution of CNTs, but also films which by their characteristics will satisfy all requirements of modern electronics. BSA showed the possibility to form stable and biocompatible composites with CNTs [23]. The concentration of BSA and SWNT was chosen from the solution maximum stability investigation.

During experiments, it was observed that in the rodcoating method the prepared films were very much homogeneous in comparison to drop-coating, and consequently the rod-coating method is preferable. Apparently, there are two major differences between the methods. Firstly, the drying time is much less in rod-coating because of a wetting. Secondly, there is a great influence of surface tension fields in drop-coating that likely leads to faster SWCNTs coagulation and bundle formation.

At Figure 2(a) we present a photograph of the film made by drop-coating, with Rs data on different points of the film. This proves the non-homogeneity of the dropcoated films. The film's topography (Figure 2(b)) has made in the $1 \mathrm{kOhm} / \mathrm{sq}$ region of the film and reveals highly percolated structure.

All the further experiments, presented data and parameters are for films made with the rod-coating method. Films prepared by this method are much more homogenous which makes them better to investigate in the first place due to adequate sheet resistances. All electrical characteristics were measured by 4-probe technique in the center of films [26-29].

\subsection{Number of Layers Effect on Resistance}

The great advantage of the rod-coating technique is achieved by spreading the solution onto a substrate surface by means of a cylindrical rod. First of all, this increases the contact surface and consequently decreases the thickness of the prepared film, thus increasing its optical transparency. Second of all, it achieved an increase in prepared film homogeneity. However, there are some drawbacks of the method related to wetting effects.

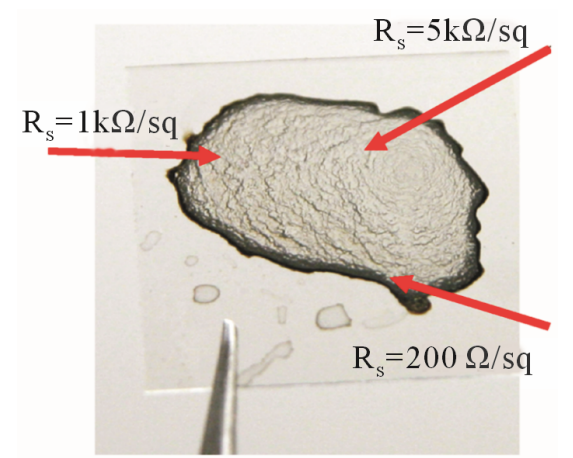

(a)

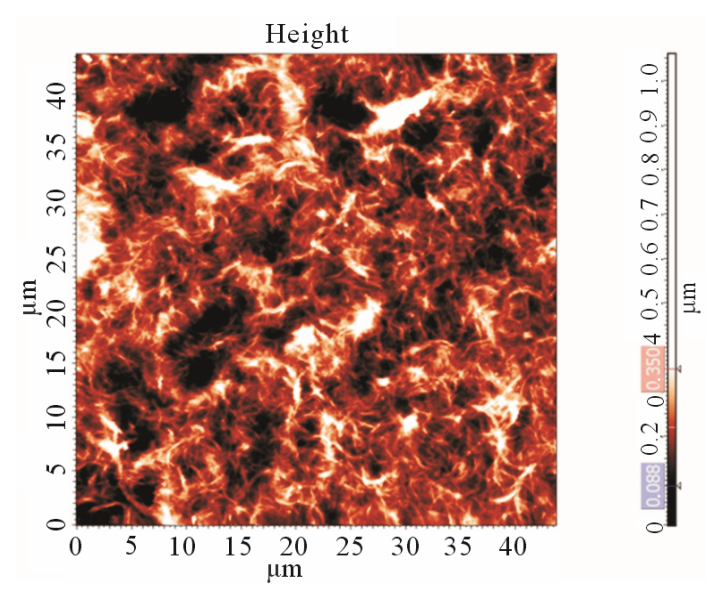

(b)

Figure 2. (a) Photograph of a CNT film made by the dropcoating method and three values of sheet resistance at different positions on the film; (b) An AFM image of as-prepared CNT film on a cover-glass (after annealing). The CNT film is highly percolated.

It was shown that, at some critical concentrations of CNTs and BSA in aqueous solution, the glass surface becomes almost impossible to wet. The wetting effect is inversely proportional to CNT and BSA concentration. So it is quite interesting that by increasing CNT and BSA concentrations in water, the wettability of glass is decreasing. In our case, the best possible concentrations were found to be 0.01 mass.\% CNT and 0.04 mass.\% BSA.The obtained data regarding optimal CNT and BSA concentrations in aqueous solution are in good agreement with results from other work [19].

Another feature of the rod-method-is the dependence of its resistivity on the number of deposited layers and the annealing technique used. In Figure 3, one can see this dependence. After annealing, film resistivity in the first two layers dramatically increase, while in the third iteration, the effect dwindles to almost nothing. It was also shown that the smaller the diameter of the rod, the more difficult it is to make completely homogeneous films. In our work, a fluorine rod with a diameter $5 \mathrm{~mm}$ was used. 


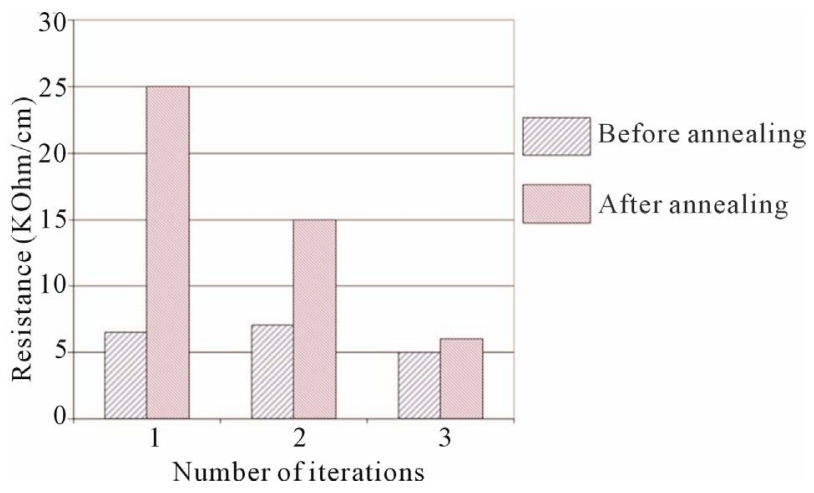

Figure 3. Effect of annealing on CNT films made by the rodcoating method by means of several iterations. Blue (left bar)_before annealing, red (right bar)—after annealing.

\subsection{Transparency and Conductivity of the Films}

Figure 4 shows spectrophotometric curves for 4 different samples, which differ only by CNT concentration in solution and consequently their different values of sheet resistance.

As it is clear from these curves, there is a direct dependence between transmission coefficient and sheet resistance. In other words, the higher the transmittance, the lower film percolation and hence the higher sheet resistance. As one can see from Figure 4, there are peaks of absorbance at $670-700 \mathrm{~nm}$, this result is in good accordance with another work which states that the breed peak of absorbance (consequently, the valley for transmission) around $670-800 \mathrm{~nm}$ indicates the existence of aggregated SWCNTs because inter-tube van der Waals interaction [30]. The peak is almost dwindles in our case in " $10 \backslash 1$ " and " $33 \backslash 1$ " solutions, because the concentration of CNTs in regards to concentration of BSA is small, therefore the film's thickness is also small and aggregation of CNTs in bundles is less favorable. Whilst at the same time, for " $1 / 1$ " and " $5 / 1$ " solutions sharp peaks indicate strong CNT aggregation in highly conductive film.

At low nanotube concentration (high BSA/CNT ratio " $10 / 1$ " and " $33 / 1$ ") the transmission is mostly defined by optic properties of BSA film. The sheet resistance for these samples can't be measured precisely because of inhomogeneity of the films. It only can be indicative that sheet resistance for the " $10 / 1$ " and " $33 / 1$ " cases is relatively high.

Based on measured values for $R_{S}$ and $T$ in our CNT films, it is possible to estimate DC conductivity- $\sigma_{d c}$ of the film by means of the following equation [31]:

$$
T=\left(1+\frac{1}{2 R_{S}} \sqrt{\frac{\mu_{0}}{\varepsilon_{0}}} \frac{\sigma_{o p}}{\sigma_{d c}}\right)^{-2}=\left(1+\frac{188(\Omega)}{R_{S}} \frac{\sigma_{o p}}{\sigma_{d c}}\right)^{-2}
$$

where $\sqrt{ }\left(\mu_{0} / \varepsilon_{0}\right)=z_{0}=377 \Omega$ is the impedance of free space and $\sigma_{o p}$ is the optical conductivity [26].

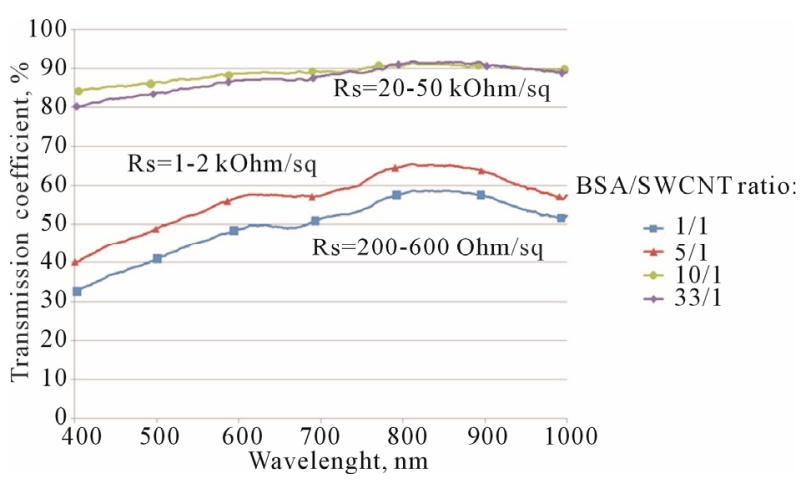

Figure 4. Vis-curves for 4 different samples, which differ only by CNT/albumin mass ratio of solution. In the case of $\mathrm{BSA} / \mathrm{SWCNT}$ ratio $1 / 1$ the average resistance is 200 - 600 $\mathrm{Ohm} /$ square; Increasing the ratio to $5 / 1$ increases the resistance to 1 - $2 \mathrm{kOhm} / \mathrm{sq}$; further increase of BSA concentration to $10 / 1$ and 33/1 leads to saturation in resistance of 20 $50 \mathrm{kOhm} / \mathrm{sq}$.

Equation (1) is commonly used for measuring the film's parameter $\sigma_{d c} / \sigma_{o p}$, if the value $\sigma_{o p}$ is known. Since the $\sigma_{o p}$ is different for different materials, we use the following for CNTs: $\sigma_{o p}=200 \mathrm{~S} \cdot \mathrm{cm}^{-1}[11,32]$.

However, the value of $\sigma_{d c}$, measured by the equation is not the true value of conductivity for every film, primarily because $\sigma_{o p}$ is different for different materials. Therefore, in the general case it is necessary to use the $\sigma_{d c} / \sigma_{o p}$ value for comparison of different films.

In order to plot the dependence between transmittance and sheet resistance (Figure 5(a)), $\sigma_{d c} / \sigma_{o p}$ and transmittance (Figure 5(b)), films with different CNT concentrations were made.

Figure 6 shows comparison of the films made in the work and films from other publications. As it is clear, our films are inferior to most of the other works, but it should be pointed out the main purpose of these work are mostly focused on getting the higher conductivity possible, and do a look into bio-compatibility of the films.

To investigate the mechanism of nanotube and BSA interaction the Raman shift measurements were carried out on 4 different samples: 1) Pure nanotubes from water solution (without BSA); 2) Pure BSA powder; 3) BSA film from an aqueous solution; and 4) The CNT-BSA water solution.

Basically, from Figure 7 it is clear that CNT spectra do not change dramatically during BSA functionalization: CNTs keep their properties. The standard peeks of protein presence at phenyl, amide I, protein side chain deformation (chain) bands. Serum albumin has disulfide bridges, and they play important roles in the intramolecular sulfhydryl-disulfide exchange reaction [33]. Information about the disulfide bridges conformation (S-S) is a matter of great importance. Of note in Figure 7 is that disulfide stretching vibrations of BSA generate a 


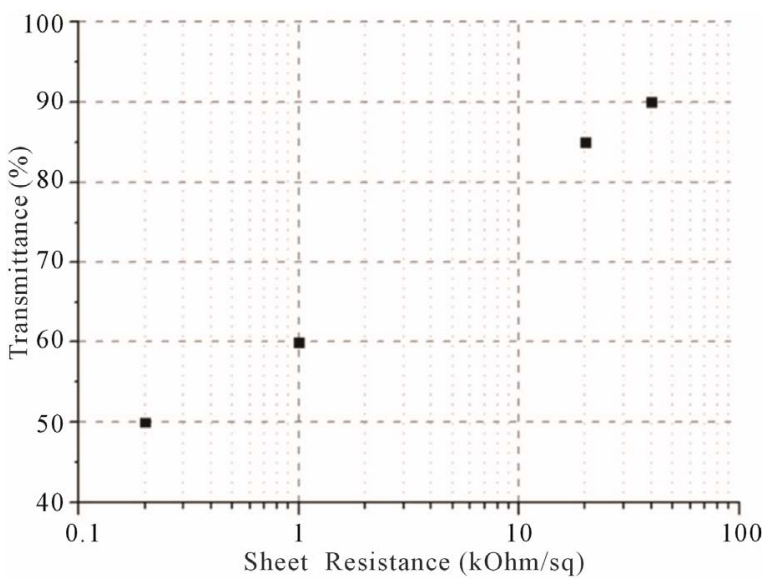

(a)

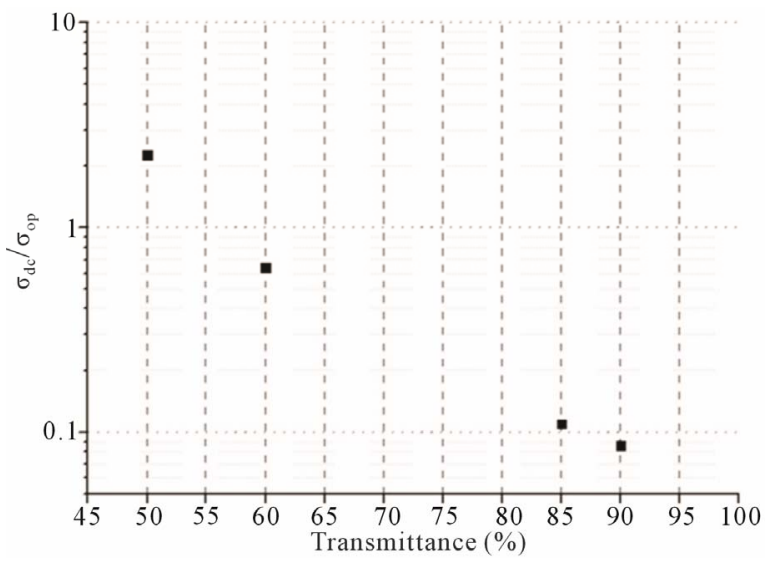

(b)

Figure 5. (a) A plot of the dependence between transmittance (at $550 \mathrm{~nm}$ wavelength) and sheet resistance (measured by 4-probe technique); (b) A plot of the dependence between transmittance (at $550 \mathrm{~nm}$ wavelength) and $\sigma_{d c} / \sigma_{o p}$.

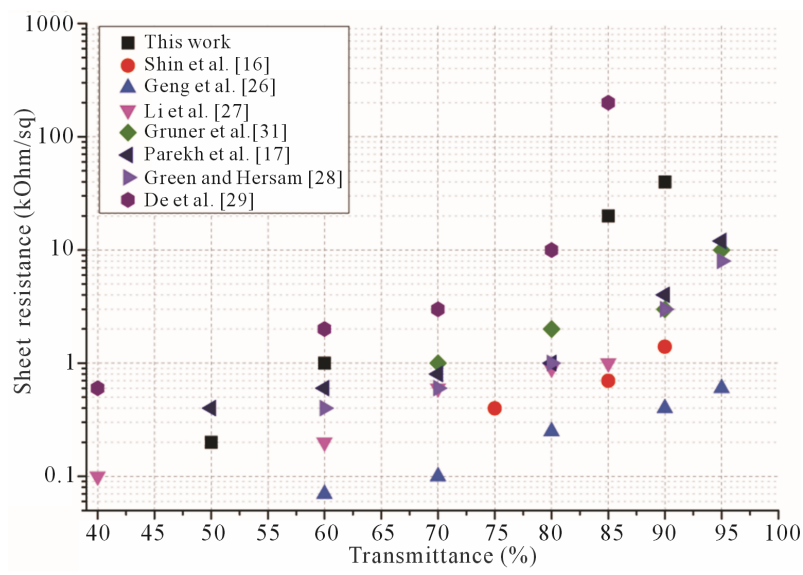

Figure 6. Comparison of dependences of sheet resistance to transmittance for different works.

strong Raman band at $517 \mathrm{~cm}^{-1}$. It can be observed that S-S band intensity remains at SWNT/BSA Raman spec-

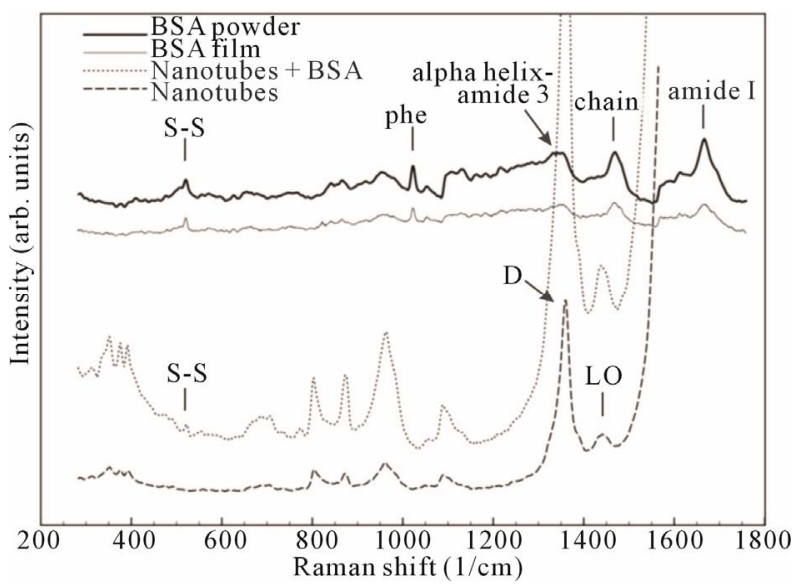

Figure 7. Comparative normalized spectral characteristics of BSA powder, BSA film, nanotubes with BSA and pure nanotubes.

tra without any change.

Raman scattering is a powerful tool that provides an investigation of the aggregate states of SWCNTs in solution. In this case, the most changes take place in the RBM mode area with the aggregate state decreasing, corresponding to a peak number decrease and an aspect ratio increase [34]. In the case of CNTs functionalized by proteins, the peaks in either RBM mode $\left(150-350 \mathrm{~cm}^{-1}\right)$ or tangential mode could be used as CNT dispersity indicators [35].

As obtained in our results, the main SWNT bands remain the same as in the films deposited from the CNTBSA aqueous solution. Nevertheless, increases in peaks intensity if observed could be evidence of increased dispersity, perhaps by ultrasonication treatments of the solution or by the penetration of albumin molecules into the CNTs surrounding area.

\subsection{Biocompatibility}

From Raman spectra it could be speculated albumin do not changes it conformation during interaction with nanotube. Biocompatibility of the films was investigated on human embryonic fibroblast (HEF). As it is clear from Figure 8, the cells grow onto the CNT films and are stable within 7 days. So, there is no toxicological effect mentioned in work because the main cause for such effect is penetration of CNTs through a cell membrane [36]. In the case of films, van der Waals interaction forces play a large role, and the lower the CNT diameter, the stronger their interaction with a surface. Therefore, SWCNTs in the film interact with the substrate, and it is favorable for them to be attached to it.

\section{Conclusions}

In summary, the method for making transparent, conduc- 


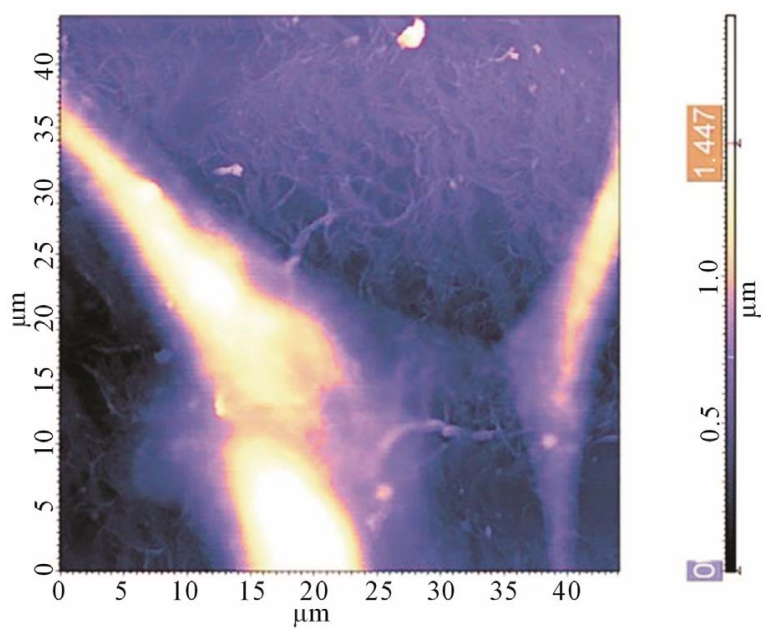

Figure 8. AFM image of a HEF cell, grown onto a CNTBSA film.

tive and biocompatible CNT-BSA films has been devel oped. We have proposed a novel method of CNT films/electrodes fabrication with using BSA as a surfactant and the rod-coating technique for films deposition. It was demonstrated, that the CNT films, obtained by the method, achieve $90 \%$ transparency with resistivity of about $45 \mathrm{kOhms} / \mathrm{square}$ and the value of $\sigma_{d c} / \sigma_{o p}=0.9$.

Nevertheless in this work the films were deposited only onto glass substrates, the method has no restrictions to be used with polymeric, flexible substrates, or even could be deposited onto a skin layer. So, this work demonstrates the potential of creating transparent, conductive and biocompatible elements, which could be used for hybrid or implanted electronic devices.

\section{Acknowledgements}

Authors are grateful to M. Simunin for critical discussion of the work.

The study was supported by The Ministry of education and science of Russian Federation, projects 14.B37.21. 1072 and 14.B37.21.0085.

\section{REFERENCES}

[1] E.-S. Choi, M.-H. Jeong, K. W. Choi, C. Lim and S.-B. Lee, "Flexible and Transparent Touch Sensor Using Single-Wall Carbon Nanotube Thin-Films," 3rd International Nanoelectronics Conference (INEC), Hong Kong, 3-8 January 2010, pp. 718-719.

[2] S. J. Wang, Y. Geng, Q. Zheng and J.-K. Kim, "Fabrication of Highly Conducting and Transparent Graphene Films," Carbon, Vol. 48, No. 6, 2010, pp. 1815-1823. doi:10.1016/j.carbon.2010.01.027

[3] J. Peltola, C. Weeks, I. A. Levitsky, D. A. Britz, P. Glatkowski, M. Trottier and T. Huang, "Carbon-Nanotube Transparent Electrodes for Flexible Displays," Information Display, Vol. 23, No. 2, 2007, pp. 20-23.
[4] D. Zhang, K. Ryu, X. Liu, E. Polikarpov, J. Ly, M. E. Tompson and C. Zhou, "Transparent, Conductive, and Flexible Carbon Nanotube Films and Their Application in Organic Light-Emitting Diodes," Nano Letters, Vol. 6, No. 9, 2006, pp. 1880-1886. doi:10.1021/n10608543

[5] B. J. Landi, R. P. Raffaelle, S. L. Castro and S. G. Bailey, "Single-Wall Carbon Nanotube-Polymer Solar Cells," Progress in Photovoltaics: Research and Applications, Vol. 13, No. 2, 2005, pp. 165-172. doi:10.1002/pip.604

[6] B. O'Neill, "Indium Market Forces, a Commercial Perspective," 35th IEEE Photovoltaic Specialists Conference (PVSC), Honolulu, 20-25 June 2010, pp. 556-560.

[7] L. Yang, T. Zhang, H. Zhou, S. C. Price, B. J. Wiley and W. You, "Solution-Processed Flexible Polymer Solar Cells with Silver Nanowire Electrodes," Applied Materials \& Interfaces, Vol. 3, No. 10, 2011, pp. 4075-4084. doi:10.1021/am2009585

[8] Y. Chen, Y. Xu, K. Zhao, X. Wan, J. Deng and W. Yan, "Towards Flexible All-Carbon Electronics: Flexible Organic Field-Effect Transistors and Inverter Circuits Using Solution-Processed All-Graphene Source/Drain/Gate Electrodes," Nano Research, Vol. 3, No. 10, 2010, pp. 714 721. doi:10.1007/s12274-010-0035-3

[9] L. Hu, D. S. Hecht and G. Gruner, "Carbon Nanotube Thin Films: Fabrication, Properties, and Applications," Chemical Reviews, Vol. 110, No. 10, 2010, pp. 5790 5844. doi:10.1021/cr9002962

[10] J. Zhang, L. Gao, J. Sun, Y. Liu, Y. Wang, J. Wang, H. Kajiura, Y. Li, and K. Noda, "Dispersion of Single-Walled Carbon Nanotubes by Nafion in Water/Ethanol for Preparing Transparent Conducting Films," The Journal of Physical Chemistry C, Vol. 112, No. 42, 2008, pp. 1637016376. doi: $10.1021 /$ jp8053839

[11] D. S. Hecht, A. M. Heintz, R. Lee, L. Hu, B. Moore, C. Cucksey and S. Risser, "High Conductivity Transparent Carbon Nanotube Films Deposited from Superacid," Nanotechnology, Vol. 22, No. 7, 2011, p. 075201. doi:10.1088/0957-4484/22/7/075201

[12] Y.-L. Zhao and J. F. Stoddart, "Noncovalent Functionalization of Single-Walled Carbon Nanotubes," Accounts of Chemical Research, Vol. 42, No. 8, 2009, pp. 1161-1171. doi:10.1021/ar900056z

[13] R. D. Breukers, K. J. Gilmore, M. Kita, K. K. Wagner, M. J. Higgins, S. E. Moulton, G. M. Clark, D. L. Officer, R. M. I. Kapsa and G. G. Wallace, "Creating Conductive Structures for Cell Growth: Growth and Alignment of Myogenic Cell Types on Polythiophenes," Journal of Biomedical Materials Research Part A, Vol. 95, No. 1, 2010, pp. 256-268. doi:10.1002/jbm.a.32822

[14] J. Xie, M. R. MacEwan, S. M. Willerth, X. Li, D. W. Moran, S. E. Sakiyama-Elbert and Y. Xia, "Conductive CoreSheath Nanofibers and Their Potential Application in Neural Tissue Engineering," Advanced Functional Materials, Vol. 19, 2009, pp. 2312-2318. doi:10.1002/adfm.200801904

[15] L. Dong, K. L. Joseph, C. M. Witkowski and M. M. Craig, "Cytotoxicity of Single-Walled Carbon Nanotubes Suspended in Various Surfactants," Nanotechnology, Vol. 19, 2008, p. 255702. doi:10.1088/0957-4484/19/25/255702 
[16] D.-W. Shin, J. H. Lee, Y.-H. Kim, S. M. Yu, S.-Y. Park and J.-B. Yoo, "A Role of $\mathrm{HNO}_{3}$ on Transparent Conducting Film with Single-Walled Carbon Nanotubes," Nanotechnology, Vol. 20, 2009, p. 47570. doi:10.1088/0957-4484/20/47/475703

[17] B. B. Parekh, G. Fanchini, G. Eda and M. Chhowalla, "Improved Conductivity of Transparent Single-Wall Carbon Nanotube Thin Films via Stable Postdeposition Functionalization," Applied Physics Letters, Vol. 90, 2007, p. 121913. doi:10.1063/1.2715027

[18] A. V. Krestinin, N. A. Kiselev, A. V. Raevskii, A. G. Ryabenko, D. N. Zakharov and G. I. Zvereva, "Perspectives of Single-Wall Carbon Nanotube Production in the Arc Discharge Process," Eurasian Chemico-Technological Journal, Vol. 5, No. 1, 2003, p. 7.

[19] S. Karajanagi, P. Asuri, J. S. Dordick and R. S. Kane, "Protein-Assisted Solubilization of Single-Walled Carbon Nanotubes," Langmuir, Vol. 22, No. 4, 2006, pp. 13921395. doi:10.1021/la0528201

[20] P. Asuri, S. S. Karajanagi, H. Yang, T. J. Yim, R. S. Kane and J. S. Dordick, "Increasing Protein Stability through Control of the Nanoscale Environment," Langmuir, Vol. 22, No. 13, 2006, pp. 5833-5836. doi:10.1021/la0528450

[21] Y. H. Yan, M. B. Chan-Park and Q. Zhang, "Advances in Carbon-Nanotube Assembly," Small, Vol. 3, 2007, pp. 2442. doi:10.1002/smll.200600354

[22] D. C. Carter and J. X. Ho, "Structure of Serum Albumin," Advances in Protein Chemistry, Vol. 45, 1994, pp. 153203. doi:10.1016/S0065-3233(08)60640-3

[23] V. M. Podgaetsky, S. V. Selishchev, I. I. Bobrinetskii and V. K. Nevolin, "Volumetric Nanodesign by New Laser Method. Application for Medical Purposes," Optical Memory \& Neural Networks, Vol. 17, No. 2, 2008, pp. 147151. doi:10.3103/S1060992X08020082

[24] T. Crouzier, A. Nimmagadda, M. U. Nollert and P. S. McFetridge, "Modification of Single Walled Carbon Nanotube Surface Chemistry to Improve Aqueous Solubility and Enhance Cellular Interactions," Langmuir, Vol. 24, No. 22, 2008, pp. 13173-13181. doi:10.1021/1a801999n

[25] M. Zheng, A. Jagota, E. D. Semke, B. A. Diner, R. S. McLean, S. R. Lustig, R. E. Richardson and N. G. Tassi, "DNA-Assisted Dispersion and Separation of Carbon Nanotubes," Nature Materials, Vol. 2, 2003, pp. 338-342. doi:10.1038/nmat877

[26] H.-Z. Geng, K. K. Kim, K. P. So, Y. S. Lee, Y. Chang and Y. H. Lee, "Effect of Acid Treatment on Carbon Nanotube-Based Flexible Transparent Conducting Films," Journal of the American Chemical Society, Vol. 129, No.
25, 2007, pp. 7758-7759. doi:10.1021/ja0722224

[27] Z. Li, H. R. Kandel, E. Dervishi, V. Saini, A. S. Biris, A. R. Biris and D. Lupu, "Does the Wall Number of Carbon Nanotubes Matter as Conductive Transparent Material?" Applied Physics Letters, Vol. 91, No. 5, 2007, Article ID: 053115. doi:10.1063/1.2767215

[28] A. A. Green and M. C. Hersam, "Processing and Properties of Highly Enriched Double-Wall Carbon Nanotubes," Nature Nanotechnology, Vol. 4, 2009, pp. 64-70. doi:10.1038/nnano.2008.364

[29] S. De, C. S. Boland, P. J. King, S. Sorel, M. Lotya, U. Patel, Z. L. Xiao and J. N. Coleman, "Transparent Conducting Films from $\mathrm{NbSe}_{3}$ Nanowires," Nanotechnology, Vol. 22, No. 28, 2011, Article ID: 285202. doi:10.1088/0957-4484/22/28/285202

[30] S. H. Hong, T. T. Tung, L. K. H. Trang, T. Y. Kim and K. S. Suh, "Preparation of Single-Walled Carbon Nanotube (SWNT) Gel Composites Using Poly (Ionic Liquids)," Colloid and Polymer Science, Vol. 288, No. 9, 2010, pp. 1013-1018. doi:10.1007/s00396-010-2229-3

[31] L. Hu, D. S. Hecht and G. Gruner, "Percolation in Transparent and Conducting Carbon Nanotube Networks," Nano Letters, Vol. 4, No. 12, 2004, pp. 2513-2517. doi:10.1021/n1048435y

[32] B. Ruzicka, L. Degiorgi, R. Gaal, L. Thien-Nga, R. Bacsa, J.-P. Salvetat and L. Forro, "Optical and dc Conductivity Study of Potassium-Doped Single-Walled Carbon Nanotube Films," Physical Review B, Vol. 61, 2000, pp. 24682471. doi:10.1103/PhysRevB.61.R2468

[33] K. Nakamura, S. Era, Y. Ozaki, M. Sogami, T. Hayashi and M. Murakami, "Conformational Changes in Seventeen Cystinedisulfide Bridges of Bovine Serum Albumin Proved by Raman Spectroscopy," FEBS Letters, Vol. 417, No. 3, 1997, pp. 375-378. doi:10.1016/S0014-5793(97)01326-4

[34] D. A. Heller, P. W. Barone, J. P. Swanson, R. M. Mayrhofer and M. S. Strano, "Using Raman Spectroscopy to Elucidate the Aggregation State of Single-Walled Carbon Nanotubes," Journal of Physical Chemistry B, Vol. 108, No. 22, 2004, pp. 6905-6909. doi:10.1021/jp037690o

[35] K. Rege and I. L. Medintz, "Methods in Bioengineering: Nanoscale Bioengineering and Nanomedicine," Artech House, London, 2009.

[36] C. M. Sayes, F. Liang and J. L. Hudson, "Functionalization Density Dependence of Single-Walled Carbon Nanotubes Cytotoxicity in Vitro," Toxicology Letters, Vol. 161, No. 2, 2006, pp. 135-142. doi:10.1016/j.toxlet.2005.08.011 